\title{
Solution to the $1+1$ dimensional gauged chiral Fermion problem
}

\author{
Juven Wang ${ }^{1,2, *}$ and Xiao-Gang Wen ${ }^{3, \dagger}$ \\ ${ }^{1}$ School of Natural Sciences, Institute for Advanced Study, Princeton, New Jersey 08540, USA \\ ${ }^{2}$ Center of Mathematical Sciences and Applications, Harvard University, Massachusetts 02138, USA \\ ${ }^{3}$ Department of Physics, Massachusetts Institute of Technology, Cambridge, Massachusetts 02139, USA
}

(Received 16 July 2018; published 10 June 2019)

\begin{abstract}
We show that the $3450 \mathrm{U}(1)$ chiral fermion theory can appear as the low energy effective field theory of a $1+1 \mathrm{D}$ local lattice model of fermions, with an on-site U(1) symmetry and finite-range interactions. The on-site U(1) symmetry means that the U(1) symmetry can be gauged (gaugeable for both background probe and dynamical fields), which leads to a nonperturbative definition of chiral gauge theory - a chiral fermion theory coupled to U(1) gauge theory. Our construction can be generalized to regularize any $\mathrm{U}(1)$-anomaly-free $1+1 \mathrm{D}$ gauged chiral fermion theory with a zero chiral central charge (thus no gravitational anomaly) by a lattice, thanks to the recently proven "Poincaré dual" equivalence between the U(1) 't Hooft anomaly-free condition and the U(1) symmetric interaction gapping rule, via a bosonizationfermionization technique.
\end{abstract}

DOI: 10.1103/PhysRevD.99.111501

The standard model [1-6] is a $\mathrm{U}(1) \times \mathrm{SU}(2) \times \mathrm{SU}(3)$ gauge theory coupled to fermions that describes all known elementary particles, but until a few years ago, the standard model was only defined perturbatively, and it is well known that such a perturbative expansion does not converge. So the standard model is a good way to fit experimental data, but itself is not a well-defined quantum theory with a finite dimensional Hilbert space for a finite size system. The reason that the standard model is not a well-defined quantum theory is because the left-hand and right-hand fermions in the standard model carry different $\mathrm{U}(1) \times \mathrm{SU}(2)$ representations. Such kinds of fermions are known as chiral fermions [7]. To define a gauged chiral fermion theory beyond perturbation is a longstanding problem.

There were many previous attempts for the gauged chiral fermion problem. The standard lattice gauge theory approach [8] fails since it cannot produce low energy gauged chiral fermions [9]. The Ginsparg-Wilson (GW) fermion approach [10] has problems since the chiral symmetry [11] is realized as a non-on-site symmetry [12-17] and, thus, is hard to gauge. The domain-wall fermion approach $[18,19]$ also has problems, since after coupling to gauge fields, the massless gauge bosons will propagate in one-higher dimension. The overlap-fermion approach [20-25] is a reformulation

\footnotetext{
juven@ias.edu xgwen@mit.edu
}

Published by the American Physical Society under the terms of the Creative Commons Attribution 4.0 International license. Further distribution of this work must maintain attribution to the author(s) and the published article's title, journal citation, and DOI. Funded by SCOAP ${ }^{3}$. of the domain-wall fermion approach and faces also some problems in a chiral gauge theory.

In the standard lattice gauge theory approach, the fermion interactions (except the gauge interaction) are ignored. In the mirror fermion approach proposed in 1986 [26-30], one started with a lattice model containing chiral fermions and a chiral conjugated mirror sector. Then, one includes proper direct interaction or boson mediated Swift-Smit interactions [31,32] trying to gap out the mirror sector completely, without breaking the gauge symmetry and without affecting the normal sector. One proposed condition to gap out the mirror sector is that there are symmetric mass terms among mirror fermions and composite mirror fermions to give all the (composite) mirror fermions a mass [26]. However, such a condition can be satisfied by U(1) anomalous $1+1 \mathrm{D}$ chiral mirror fermions which can never be fully gapped (see the arXiv version of Ref. [15]). This means the [26]'s criteria is not sufficient enough to produce fully gapped mirror fermions. The follow-up work [33-36] failed to demonstrate that interactions can gap out the mirror sector without breaking the symmetry in some mirror fermion models. It was argued that "attempts to decouple lattice fermion doubles by the method of Swift and Smit cannot succeed" [37] and many people gave up the mirror fermion approach.

In 2013, Ref. [14] pointed out that quantum anomalies are directly connected to and realized at the boundary of topological orders [38] or symmetry protected topological orders $[12,13,39]$ on a lattice in one higher dimension. This leads to a classification of anomalies [14,40]. From this point of view, the anomaly-free condition is nothing but the condition for the bulk to be a trivial tensor product state. This leads to a solution of the gauged chiral fermion 
problem claimed by Ref. [15]: For any chiral gauge theory that is free of "all anomalies," there exists a local lattice model of the same dimension whose low energy effective theory realizes the chiral gauge theory. Here a chiral gauge theory is defined as a boson/fermion theory coupled to gauge fields in a weak coupling limit. To actually use the above result, Ref. [15] proposed a sufficient condition: A gauged chiral fermion theory in $d$-dimensional space-time with a gauge group $G$ is free of all anomalies if (1) there exist (possibly gauge symmetry breaking) mass terms that make all the fermions massive, and (2) $\pi_{n}\left(G / G_{\text {grnd }}\right)=0$ for $0 \leq n \leq d+1$, where $G_{\text {grnd }}$ is the unbroken gauge symmetry group.

The above result implies that the $\mathrm{SO}(10)$ gauged chiral fermion theory in the $\mathrm{SO}(10)$ grand unification [41] can be regulated by a $3+1 \mathrm{D}$ lattice. This way, we solve a longstanding problem of defining the standard model nonperturbatively [15], at least for a version of standard model from an $\mathrm{SO}(10)$ grand unification. Even certain anomalous chiral gauge theories can be put on a lattice of the same dimension [14]. This new way to gap out the mirror sector was later referred as "mass without mass terms"- a new mechanism beyond the Higgs mechanism to generate mass [16,42-47]. However, the above claim has not been accepted by many people.

In this work, we will provide a supporting evidence that anomaly-free gauged chiral fermion theories can indeed be regularized on the lattice if we allow lattice fermions to interact properly. In particular, we show that the following $1+1 \mathrm{D}$ chiral fermion field theory with $\mathrm{U}(1)$ symmetry:

$$
\mathcal{L}=\sum_{a=1}^{4} \psi_{a}^{\dagger}\left(\mathrm{i} \partial_{t}-\mathrm{i} v_{a} \partial_{x}\right) \psi_{a},
$$

can be realized by a $1+1 \mathrm{D}$ lattice model with on-site $\mathrm{U}(1)$ symmetry in low energies [16,47]. Here $a=1,2,3,4$ and $\left(v_{1}, v_{2}, v_{3}, v_{4}\right)=(1,1,-1,-1)$. Namely, we have two leftmoving Weyl fermions $\psi_{1}$ and $\psi_{2}$ with charge 3 and 4 , and two right-moving Weyl fermions $\psi_{3}$ and $\psi_{4}$ with charge 5 and 0 . We refer the above $\mathrm{U}(1)$ chiral fermion theory as the 3450 theory. We note that the gauged 3450 chiral fermion theory does not satisfy the above sufficient condition, but below we show the mechanism of "mass without mass terms" still works.

The $1+1 \mathrm{D}$ lattice quantum Hamiltonian model that realized the 3450 chiral fermion theory is given by

$$
\hat{H}=\sum_{a, i}\left(\frac{\mathrm{i}}{2} v_{a} \hat{c}_{a, i}^{\dagger} \hat{c}_{a, i+1}+\text { H.c. }\right)+\hat{H}_{\mathrm{int}},
$$

where $\hat{H}_{\text {int }}$ describes the short range interaction whose form will be given later. Here the lattice spacing is chosen to be 1. The $\mathrm{U}(1)$ charges of the lattice fermion operators $\hat{c}_{1}, \hat{c}_{2}, \hat{c}_{3}, \hat{c}_{4}$ are given by $\left(q_{1}, q_{2}, q_{3}, q_{4}\right)=(3,4,5,0)$ and the lattice model has an on-site $\mathrm{U}(1)$ symmetry: $\hat{c}_{a, i} \rightarrow \mathrm{e}^{\mathrm{i} q_{a} \theta} \hat{c}_{a, i}$. (An on-site symmetry is a special global symmetry that is a tensor product of symmetry transformations on each site. Global symmetries on the lattice with 't Hooft anomalies are necessarily non-on-site [12-17].) In this paper, we will show that, after choosing the interaction $\hat{H}_{\text {int }}$ properly, the lattice model (2) will produce the chiral fermion theory (1) at low energies. This is the key result of the paper.

In the lattice model (2), we do not use GW fermion at all, thus we do not encounter the difficulty of gauging GW's non-on-site symmetry [16,17]. Our model (2) has an on-site $\mathrm{U}(1)$ symmetry, which can be easily gauged on a lattice, to obtain a fermionic lattice model coupled to a U(1) lattice gauge field with a lattice Lagrangian $L_{\text {latt }}$ :

$$
\begin{aligned}
L_{\mathrm{latt}}= & \sum_{i, a} c_{a, i}^{\dagger}\left(\mathrm{i} \partial_{t}+q_{a} A_{i, 0}\right) c_{a, i} \\
& -\sum_{i, a}\left(\frac{\mathrm{i}}{2} v_{a} c_{a, i}^{\dagger} c_{a, i+1} \mathrm{e}^{\mathrm{i} q_{a} A_{i, i+1}}+\text { H.c. }\right)-H_{\text {int }}(A) .
\end{aligned}
$$

Now the Lagrangian contains the fermions as Grassmann variables. The $A_{i, 0}$ is a continuous time-component potential, while the $A_{i, i+1}$ is a spatial-component gauge field on the discretized link. The $H_{\text {int }}(A)$ is the gauged version of $H_{\text {int }}$ based on the standard procedure of gauging the on-site $\mathrm{U}(1)$ symmetry by inserting gauge field $A$ on the linkswhich $H_{\text {int }}$ will be obtained later in Eq. (17). Such a lattice model will produce the gauged chiral fermion theory at low energies. This way, we show that the gauged chiral fermion theory can be defined nonperturbatively via a lattice model.

We would like to remark that it is well known that to put the gauged chiral fermion theory on a lattice, the gauge field does not need to be dynamical. The gauge field can be a fixed background gauge field. This is the point of view taken by this paper: The U(1) gauge field $A_{\mu}$ is a fixed background gauge field, and we often fix this background to be $A_{\mu}=0$. This is why the $\mathrm{U}(1)$ gauge field is often not explicit in this paper.

When $\hat{H}_{\text {int }}=0$, the low energy effective field theory of the above lattice model of free fermions is given by

$\mathcal{L}=\sum_{a=1}^{4} \psi_{a}^{\dagger}\left(\mathrm{i} \partial_{t}-\mathrm{i} v_{a} \partial_{x}\right) \psi_{a}+\psi_{M, a}^{\dagger}\left(\mathrm{i} \partial_{t}+\mathrm{i} v_{a} \partial_{x}\right) \psi_{M, a}+\cdots$,

where the chiral fermions $\psi_{a}$ correspond to lattice fermions near crystal momentum $k=\pi$, and the mirror fermions $\psi_{M, a}$ correspond to lattice fermions near crystal momentum $k=0$. Here the $\cdots$ represent the term involving high energy modes. With the $\cdots$ term, the continuum field theory (3) has the same symmetry as the lattice model (2). We would like to stress that the above effective field 
theory has a momentum cut-off $\Lambda$ that is of the same order as the inverse lattice spacing (such as $\Lambda=\frac{\pi}{4}$ ). Integrating out lattice fermions beyond the cut-off scale does not change low energy effective field theory since, by design [see Eq. (17) and the discussion following it], the lattice fermions with momentum far away from $k=0, \pi$ are noninteracting.

Eq. (3) is not a chiral fermion theory since it contains both normal sector (the chiral fermions sector) $\psi_{a}$ and the mirror sector $\psi_{M, a}$. Next we will try to gap out the mirror sector without breaking the on-site U(1) symmetry and without affecting the normal sector, by introducing interactions that affect only the mirror sector. Let us first describe the required interaction within the effective field theory (3).

Using the null-vector condition [48-50] from quantum Hall edge states [51,52] (for a more general discussion, see Refs. [53-55]), we can show that, by choosing a proper interaction within the mirror sector, we can indeed gap out all the mirror fermions $\psi_{M, a}$, without breaking the U(1) symmetry. To see how to gap out the mirror fermions, we first bosonize them by introducing eight boson fields $\phi_{a}$ and $\phi_{M, a}, a=1, \ldots, 4$ :

$$
: \mathrm{e}^{\mathrm{i} \phi_{a}}:=\psi_{a}, \quad: \mathrm{e}^{\mathrm{i} \phi_{M, a}}:=\psi_{M, a} .
$$

Note that the value of the boson fields is only defined modular $2 \pi$. Here the mapping between operators needs to be careful with a proper normal ordering $\left(: \mathrm{e}^{\mathrm{i} \phi}:\right)$ on the bosonic operator side, following the standard bosonization procedure. However, the mapping between the whole Lagrangians is more straightforward. The bosonized low energy effective field theory is described by (see, for example, Ref. [56].)

$$
\begin{aligned}
\mathcal{L}= & \frac{1}{4 \pi}\left(K_{a b} \partial_{t} \phi_{a} \partial_{x} \phi_{b}-\partial_{x} \phi_{a} \partial_{x} \phi_{a}\right) \\
& +\frac{1}{4 \pi}\left(-K_{a b} \partial_{t} \phi_{M, a} \partial_{x} \phi_{M, b}-\partial_{x} \phi_{M, a} \partial_{x} \phi_{M, a}\right),
\end{aligned}
$$

where $K$ is diagonal with $\operatorname{diag}(K)=(1,1,-1,-1)$. Now we can introduce an interaction

$$
\begin{aligned}
\mathcal{L}_{\text {int }}= & \pi \Delta V_{a b} \rho_{M, a} \rho_{M, b}+\tilde{g}_{1} \psi_{M, 1}\left(\psi_{M, 2}^{\dagger}\right)_{p t . s}^{2} \psi_{M, 3}\left(\psi_{M, 4}\right)_{p t . s}^{2} \\
& +\tilde{g}_{2}\left(\psi_{M, 1}^{\dagger}\right)_{p t . s}^{3} \psi_{M, 2} \psi_{M, 3}\left(\psi_{M, 4}^{\dagger}\right)_{p t . s}^{3} \\
= & \frac{1}{4 \pi} \Delta V_{a b} \partial_{x} \phi_{M, a} \partial_{x} \phi_{M, b} \\
& +g_{1} \cos \left(l_{1, a} \phi_{M, a}\right)+g_{2} \cos \left(l_{2, a} \phi_{M, a}\right)
\end{aligned}
$$

where $\rho_{M, a}=\psi_{M, a}^{\dagger} \psi_{M, a}=\frac{1}{2 \pi} \partial_{x} \phi_{M, a}$ is the density of the mirror fermions, the fermion point splitting (pt.s) is defined as higher derivative term: $\left(\psi_{M, a}\right)_{p t . s}^{n} \equiv \psi_{M, a}$ $\left(\partial_{x} \psi_{M, a}\right) \ldots\left(\partial_{x}^{n-1} \psi_{M, a}\right)$, and

$$
\boldsymbol{l}_{1}=(1,-2,1,2), \quad \boldsymbol{l}_{2}=(-3,1,1,-3) .
$$

After we include the interaction $\mathcal{L}_{\text {int }}$, the bosonized low energy effective Lagrangian becomes

$$
\begin{aligned}
\mathcal{L}= & \frac{1}{4 \pi}\left(-K_{a b} \partial_{t} \phi_{M, a} \partial_{x} \phi_{M, b}-V_{a b} \partial_{x} \phi_{M, a} \partial_{x} \phi_{M, b}\right) \\
& +g_{1} \cos \left(l_{1, a} \phi_{M, a}\right)+g_{2} \cos \left(l_{2, a} \phi_{M, a}\right) \\
& +\frac{1}{4 \pi}\left(K_{a b} \partial_{t} \phi_{a} \partial_{x} \phi_{b}-\partial_{x} \phi_{a} \partial_{x} \phi_{a}\right),
\end{aligned}
$$

where $V_{a b}=\delta_{a b}+\Delta V_{a b}$, and $g_{i}$ is given by $\tilde{g}_{i}$ times a factor that depends on the cutoff. We note that $\boldsymbol{l}_{1}, \boldsymbol{l}_{2}$ are chosen such that $\mathcal{L}_{\text {int }}$ has the U(1) symmetry. $\boldsymbol{l}_{1}, \boldsymbol{l}_{2}$ also satisfy the null-vector condition [48-50,55]

$$
\boldsymbol{l}_{1}^{\top} K^{-1} \boldsymbol{l}_{1}=\boldsymbol{l}_{2}^{\top} K^{-1} \boldsymbol{l}_{2}=\boldsymbol{l}_{1}^{\top} K^{-1} \boldsymbol{l}_{2}=0,
$$

which makes the term $g_{1} \cos \left(l_{1, a} \phi_{M, a}\right)+g_{2} \cos \left(l_{2, a} \phi_{M, a}\right)$ being able to gap out the mirror sector.

In the following, we would like to discuss how to choose a proper $V_{a b}, g_{1}, g_{2}$ in Eq. (8) to gap out the mirror sector $[48-50,55]$. To understand the dynamics of the interacting mirror fermions and why the mirror fermions can all be gapped out by the interaction $\mathcal{L}_{\text {int }}$, we change the basis for the $\phi_{M, a}$ field, $\tilde{\phi}_{a}=W_{a b} \phi_{M, b}$, using the following $\mathrm{GL}(4, \mathbb{Z})$ transformation:

$$
W=\left(\begin{array}{cccc}
1 & -2 & 1 & 2 \\
0 & -3 & 2 & 2 \\
-5 & 2 & 2 & -5 \\
3 & -4 & 1 & 5
\end{array}\right)
$$

Note that the value of the new boson fields $\tilde{\phi}_{a}$ is still only defined modular $2 \pi$. In terms of $\tilde{\phi}_{a}$, the bosonized theory can be rewritten as

$$
\begin{aligned}
\mathcal{L}= & \frac{1}{2 \pi} \partial_{t} \tilde{\phi}_{1} \partial_{x} \tilde{\phi}_{3}+g_{1} \cos \left(\tilde{\phi}_{1}\right)-\frac{1}{4 \pi} \tilde{V}_{a b} \partial_{x} \tilde{\phi}_{a} \partial_{x} \tilde{\phi}_{b} . \\
& +\frac{1}{4 \pi}\left(\partial_{t} \tilde{\phi}_{4} \partial_{x} \tilde{\phi}_{4}-\partial_{t} \tilde{\phi}_{2} \partial_{x} \tilde{\phi}_{2}\right)+g_{2} \cos \left(\tilde{\phi}_{2}-\tilde{\phi}_{4}\right),
\end{aligned}
$$

where $\tilde{V}=\left(W^{-1}\right)^{\top} V W^{-1}$.

We can choose $V_{a b}$ such that $\tilde{V}_{a b}=V_{0} \delta_{a b}$. Now $\left(\tilde{\phi}_{1}, \tilde{\phi}_{3}\right)$ and $\left(\tilde{\phi}_{2}, \tilde{\phi}_{4}\right)$ decouple.

To understand the dynamics of $\left(\tilde{\phi}_{1}, \tilde{\phi}_{3}\right)$, we can integrate out $\tilde{\phi}_{3}$ since it is quadratic, and obtain

$$
\mathcal{L}=\frac{1}{4 \pi V_{0}}\left(\partial_{t} \tilde{\phi}_{1}\right)^{2}-\frac{V_{0}}{4 \pi}\left(\partial_{x} \tilde{\phi}_{1}\right)^{2}+g_{1} \cos \left(\tilde{\phi}_{1}\right) .
$$

This is the standard sine-Gordon theory and a well-known gapped phase of Luttinger liquid. Since the operator 
$\cos \left(\tilde{\phi}_{1}\right)$, having a scaling dimension $1 / 2$, is relevant, $\left(\tilde{\phi}_{1}, \tilde{\phi}_{3}\right)$ are completely gapped when $g_{1} \neq 0$. The energy gap scales as $\Delta \sim\left|g_{1}\right|^{2 / 3}$.

The dynamics of $\left(\tilde{\phi}_{2}, \tilde{\phi}_{4}\right)$ is described by

$$
\begin{aligned}
\mathcal{L}= & \frac{1}{4 \pi}\left(\partial_{t} \tilde{\phi}_{4} \partial_{x} \tilde{\phi}_{4}-\partial_{t} \tilde{\phi}_{2} \partial_{x} \tilde{\phi}_{2}\right)+g_{2} \cos \left(\tilde{\phi}_{2}-\tilde{\phi}_{4}\right) \\
& -\frac{V_{0}}{4 \pi}\left(\partial_{x} \tilde{\phi}_{2} \partial_{x} \tilde{\phi}_{2}+\partial_{x} \tilde{\phi}_{4} \partial_{x} \tilde{\phi}_{4}\right),
\end{aligned}
$$

which is the bosonized free fermion theory:

$$
\begin{aligned}
\mathcal{L}= & \tilde{\psi}_{L}^{\dagger}\left(\mathrm{i} \partial_{t}-\mathrm{i} V_{0} \partial_{x}\right) \tilde{\psi}_{L}+\tilde{\psi}_{R}^{\dagger}\left(\mathrm{i} \partial_{t}+\mathrm{i} V_{0} \partial_{x}\right) \tilde{\psi}_{R} \\
& +g_{2}\left(\tilde{\psi}_{R}^{\dagger} \tilde{\psi}_{L}+\text { H.c. }\right) .
\end{aligned}
$$

$\left(\tilde{\phi}_{2}, \tilde{\phi}_{4}\right)$ are gapped out by the fermion mass term $g_{2}\left(\tilde{\psi}_{R}^{\dagger} \tilde{\psi}_{L}+\right.$ H.c. $)$ when $g_{2} \neq 0$. The energy gap scales as $\Delta \sim\left|g_{2}\right|$. We see that the fermion interaction $\mathcal{L}_{\text {int }}$ can indeed gap out all the massless modes described by $\phi_{a}$, provided that we choose $V_{a b}$ properly.

Next, we like to show that the interaction term $\mathcal{L}_{\text {int }}$ can be realized by an interaction $\hat{H}_{\text {int }}$ on a lattice. The key is to introduce lattice fermion operators,

$$
\hat{\tilde{c}}_{a, i}=\sum_{j} f(i-j) \hat{c}_{a, j}
$$

that have a unit overlap with the fermions in the mirror sector and almost no overlap with fermions in the normal sector. This can be easily done since the fermions in the mirror sector carry $k=0$ crystal momentum, and the fermions in the normal sector carry $k=\pi$ crystal momentum. For example, we can choose the function $f(i)$ such that its Fourier transformation is given by

$$
\tilde{f}(k) \equiv \sum_{i} \mathrm{e}^{\mathrm{i} k i} f(i)=\cos ^{2 n}\left(\frac{k}{2}\right) .
$$

Such a function satisfies $f(i)=0$ when $|i|>n$, and thus has a finite range. Using $\hat{\tilde{c}}_{a, i}$, we can design the interaction Hamiltonian as

$$
\begin{aligned}
\hat{H}_{\mathrm{int}}= & \sum_{i} \pi \Delta V_{a b} \hat{\tilde{\tilde{\rho}}}_{a, i} \hat{\tilde{\tilde{\rho}}}_{b, i}+\sum_{i}\left(g_{1} \hat{\tilde{c}}_{1, i}\left(\hat{\tilde{c}}_{2, i}^{\dagger}\right)^{2} \hat{\tilde{c}}_{3, i} \hat{\tilde{c}}_{4, i}^{2}\right. \\
& \left.+g_{2}\left(\hat{\tilde{c}}_{1, i}^{\dagger}\right)^{3} \hat{\tilde{c}}_{2, i} \hat{\tilde{c}}_{3, i}\left(\hat{\tilde{c}}_{4, i}^{\dagger}\right)^{3}+\text { H.c. }\right),
\end{aligned}
$$

where $\hat{\tilde{\rho}}_{a, i}=\hat{\tilde{c}}_{a, i}^{\dagger} \hat{\tilde{c}}_{a, i}$ is the density operator of the mirror fermions, and $\hat{\tilde{c}}_{i}^{2}, \hat{\tilde{c}}_{i}^{3}$ are defined again by the point splitting $\hat{\tilde{c}}_{i}^{2} \equiv \hat{\tilde{c}}_{i} \hat{\tilde{c}}_{i+1}, \hat{\tilde{c}}_{i}^{3} \equiv \hat{\tilde{c}}_{i-1} \hat{\tilde{c}}_{i} \hat{\tilde{c}}_{i+1}$, etc. We note that the lattice interaction $\hat{H}_{\text {int }}$ involves only the mirror fermions with crystal momentum $k \sim 0$. $\hat{H}_{\text {int }}$ hardly involves any fermions in the normal sector with $k \sim \pi$ and hardly involves any fermions beyond $k$-cut-off $\Lambda>\frac{\pi}{4}$ if $n$ is not too small.
So the lattice fermions beyond cutoff remain almost noninteracting. Integrating out those fermions gives us low energy effective field theory Eq. (8), where the mirror sector is shown to be fully gapped if we choose $V_{a b}, g_{1}, g_{2}$ properly. This way, we show that 3450 chiral fermion theory can be realized at low energies of a $1+1 \mathrm{D}$ lattice model with a on-site U(1) symmetry. In other words, the chiral fermion theory (1) can be fully regularized via a lattice model (2) as its low energy effective field theory.

We remark that the gapped phases of the sine-Gordon theory (12) and the free fermion theory (13) can have correlation lengths much bigger than the cut-off length when $g_{1}, g_{2}$ are small. Therefore, the above field theory analysis of the gapping process is self-consistent. Namely, we can use the bosonized field theory to understand the gapping process of the lattice mirror fermions.

We can show that there is no additional topological ground state degeneracy (GSD) [50] from the gapped mirror sector, thus GSD $=1$ [ the energy spectrum within a tiny order $O\left(e^{-L}\right)$ for a system size $L$ ]. The only low lying modes are from the gapless chiral sector [the dense energy spectra with a small subgap $O(1 / L)]$.

The numerical calculation of Ref. [36] for a particular lattice model with Yukawa interactions fails to realize the 3450 chiral fermion theory. However, this does not exclude the possibility that a more carefully designed $1+1$ D lattice model can realize the 3450 chiral fermion theory. In particular, to realize a chiral fermion theory, the energy scale of interactions should be comparable with the kinetic term $[15,16,47]$ (instead of much bigger than the kinetic term chosen in Ref. [36]).

Our approach can be generalized to put any anomaly-free $1+1 \mathrm{D} U(1)$ chiral fermion theory on a lattice. This is because Ref. [16] proves nonperturbatively that the U(1)anomaly-free condition with zero chiral central charge $c_{L}-c_{R}=0$ (thus no gravitational anomaly) is equivalent to the U(1) symmetric interaction gapping rule. Ref. [16]'s proof is based on the compatibility of the anomaly-free condition and gapping rule [50] under the Narain lattice level quantization [57] in the context of chiral boson and Chern-Simons theories. For the number of left and right $1+1 \mathrm{D}$ Weyl fermions equal $N_{L}=N_{R}=N$, we have constructed the $\left(\mathrm{U}(1)^{N}\right)_{\text {anomaly free }}^{\text {t }}$ and $\left(\mathrm{U}(1)^{N}\right)_{\text {gapping term }}$ sectors via a short exact sequence in [16]:

$$
\left(\mathrm{U}(1)^{N}\right)_{\text {anomaly free }}^{\text {t Hooft }} \rightarrow \mathrm{U}(1)^{2 N} \rightarrow\left(\mathrm{U}(1)^{N}\right)_{\text {gapping term }} .
$$

The $\left(\mathrm{U}(1)^{N}\right)^{\prime}$ anoomaly free is the maximal torus group which carries the anomaly-free chiral $U(1)^{N}$ symmetry. The $\left(\mathrm{U}(1)^{N}\right)_{\text {gapping term }}$ is the symmetry-breaking group as the Poincaré dual $U(1)^{N}$ symmetry within the total group $\mathrm{U}(1)^{2 N}$. The $\left(\mathrm{U}(1)^{N}\right)_{\text {gapping term }}$ corresponds to both the maximal and the minimal set of nonperturbative interaction terms ( $N$-linear independent cosine terms, as Eq. (6), in 
which we have $N=2$; whose mathematical concept behind is the so-called Lagrangian subgroup/submanifold explained in [16]) to be included in the mirror sector in order to gap the mirror fermions. In our $N=2$ case, we have $\left(\mathrm{U}(1)^{2}\right)_{\text {anomaly }}^{\text {t Hooft }}$ generated by two charge vectors $\left(q_{1}, q_{2}, q_{3}, q_{4}\right)=(3,4,5,0)$ and $(0,5,4,3)$; and $\left(\mathrm{U}(1)^{2}\right)_{\text {gapping term }}$ generated by another two vectors $\boldsymbol{l}_{1}=(1,-2,1,2), \boldsymbol{l}_{2}=(-3,1,1,-3)$ in (7). Therefore, we can always realize a U(1)-anomaly-free chiral matter theory with a zero chiral central charge by a truly local $1+1 \mathrm{D}$ interacting lattice model with an on-site $\mathrm{U}(1)$ symmetry [such as Eq. (2)/(17)].

Although we describe our approach using a lattice Hamiltonian formalism, the result can be applied to the lattice Euclidean path integral formalism (for Monte Carlo simulation), as long as a proper interaction (17) is chosen [16]. In Ref. [16], via a rigorous $1+1 \mathrm{D}$ bosonizationfermionization method, we had performed the exact mapping between the bosonized theory with the sine-Gordon interaction cosine terms, and the fermionized theory with the higher-derivative multifermion interaction terms, in the continuum field theories and in the regularized lattice models.

As a well-defined quantum theory, our lattice model (2) has a well defined UV-complete fermion Green's function. Although we did not compute this interacting fermion Green's function on the lattice, such a fermion Green's function becomes the Green's function of noninteracting free chiral fermions with $3450 \mathrm{U}(1)$ chiral symmetry in Eq. (1) at low energies.

\section{ACKNOWLEDGMENTS}

J.W. thanks Edward Witten for remarks and thanks Jordan Cotler et al. for discussions. J. W. gratefully acknowledges the support of Corning Glass Works Foundation Fellowship and NSF Grant No. PHY1606531. X.-G Wen is partially supported by NSF Grants No. DMR-1506475 and No. DMS-1664412. This work is also supported by NSF Grant No. DMS-1607871 "Analysis, Geometry and Mathematical Physics" and Center for Mathematical Sciences and Applications at Harvard University.
[1] S. L. Glashow, Nucl. Phys. 22, 579 (1961).

[2] S. Weinberg, Phys. Rev. Lett. 19, 1264 (1967).

[3] A. Salam and J. C. Ward, Phys. Lett. 13, 168 (1964).

[4] M. Gell-Mann, Phys. Rev. 125, 1067 (1962).

[5] G. Zweig, in Developments In The Quark Theory Of Hadrons, edited by D. B. Lichtenberg and S.P. Rosen (Hadronic Press, Nonantum, MA, 1980), Vol. 1, p. 24.

[6] H. Fritzsch and M. Gell-Mann, in Proceedings of the XVI International Conference on High Energy Physics, Chicago, edited by J. D. Jackson and A. Roberts (Department of Energy, United States, 1972), Vol. 2, p. 135.

[7] T. D. Lee and C.-N. Yang, Phys. Rev. 104, 254 (1956).

[8] J. B. Kogut, Rev. Mod. Phys. 51, 659 (1979).

[9] H. B. Nielsen and M. Ninomiya, Phys. Lett. 105B, 219 (1981).

[10] P. Ginsparg and K. Wilson, Phys. Rev. D 25, 2649 (1982).

[11] M. Lüscher, Phys. Lett. B 428, 342 (1998).

[12] X. Chen, Z.-X. Liu, and X.-G. Wen, Phys. Rev. B 84, 235141 (2011).

[13] X. Chen, Z.-C. Gu, Z.-X. Liu, and X.-G. Wen, Phys. Rev. B 87, 155114 (2013).

[14] X.-G. Wen, Phys. Rev. D 88, 045013 (2013).

[15] X.-G. Wen, Chin. Phys. Lett. 30, 111101 (2013).

[16] J. Wang and X.-G. Wen,arXiv:1307.7480.

[17] J. Wang, X.-G. Wen, and E. Witten, Phys. Rev. X 8, 031048 (2018).

[18] D. B. Kaplan, Phys. Lett. B 288, 342 (1992).

[19] Y. Shamir, Nucl. Phys. B406, 90 (1993).

[20] R. Narayanan and H. Neuberger, Phys. Lett. B 302, 62 (1993).
[21] R. Narayanan and H. Neuberger, Nucl. Phys. B412, 574 (1994).

[22] M. Lüscher, Nucl. Phys. B549, 295 (1999).

[23] H. Neuberger, Phys. Rev. D 63, 014503 (2000).

[24] H. Suzuki, Prog. Theor. Phys. 101, 1147 (1999).

[25] M. Lüscher, Subnucl. Ser. 38, 41 (2002).

[26] E. Eichten and J. Preskill, Nucl. Phys. B268, 179 (1986).

[27] I. Montvay, Nucl. Phys. B, Proc. Suppl. 29, 159 (1992).

[28] T. Bhattacharya, M. R. Martin, and E. Poppitz, Phys. Rev. D 74, 085028 (2006).

[29] J. Giedt and E. Poppitz, J. High Energy Phys. 10 (2007) 76.

[30] E. Poppitz and Y. Shang, Int. J. Mod. Phys. A 25, 2761 (2010).

[31] P. D. V. Swift, Phys. Lett. 145B, 256 (1984).

[32] J. Smit, Acta Phys. Pol. B 17, 531 (1986).

[33] M. Golterman, D. Petcher, and E. Rivas, Nucl. Phys. B395, 596 (1993).

[34] L. Lin, Phys. Lett. B 324, 418 (1994).

[35] M. F. L. Golterman and Y. Shamir, Phys. Rev. D 51, 3026 (1995).

[36] C. Chen, J. Giedt, and E. Poppitz, J. High Energy Phys. 131 (2013) 1304.

[37] T. Banks and A. Dabholkar, Phys. Rev. D 46, 4016 (1992).

[38] X.-G. Wen, Int. J. Mod. Phys. B 04, 239 (1990).

[39] Z.-C. Gu and X.-G. Wen, Phys. Rev. B 80, 155131 (2009).

[40] L. Kong and X.-G. Wen,arXiv:1405.5858.

[41] H. Fritzsch and P. Minkowski, Ann. Phys. (N.Y.) 93, 193 (1975).

[42] Y.-Z. You, Y. BenTov, and C. Xu, arXiv:1402.4151.

[43] Y.-Z. You and C. Xu, Phys. Rev. B 91, 125147 (2015). 
[44] Y. BenTov and A. Zee, Phys. Rev. D 93, 065036 (2016).

[45] V. Ayyar and S. Chandrasekharan, Phys. Rev. D 93, 081701 (2016).

[46] V. Ayyar and S. Chandrasekharan, J. High Energy Phys. 10 (2016) 58.

[47] M. DeMarco and X.-G. Wen, arXiv:1706.04648.

[48] F. D. M. Haldane, Phys. Rev. Lett. 74, 2090 (1995).

[49] H.-C. Kao, C.-H. Chang, and X.-G. Wen, Phys. Rev. Lett. 83, 5563 (1999).

[50] J. Wang and X.-G. Wen, Phys. Rev. B 91, 125124 (2015).
[51] B. I. Halperin, Phys. Rev. B 25, 2185 (1982).

[52] X.-G. Wen, Phys. Rev. B 41, 12838 (1990).

[53] A. Kapustin and N. Saulina, Nucl. Phys. B845, 393 (2011).

[54] A. Kitaev and L. Kong, Commun. Math. Phys. 313, 351 (2012).

[55] M. Levin, Phys. Rev. X 3, 021009 (2013).

[56] X.-G. Wen, Adv. Phys. 44, 405 (1995).

[57] K. S. Narain, M. H. Sarmadi, and E. Witten, Nucl. Phys. B279, 369 (1987). 